\title{
CONF-941129--28
}

\section{Nuclear Microscopy of Sperm Cell \\ Elemental Structure}

Graham S. Bench, Rodney Balhorn, Alexander M. Friz and Stewart P.H.T. Freeman

\section{RECEIVED APR 051996}

This paper was prepared for submission to the 13th International Conference on the Applications of Accelerators in Research and Industry

November 7-10, 1994

September 28, 1994

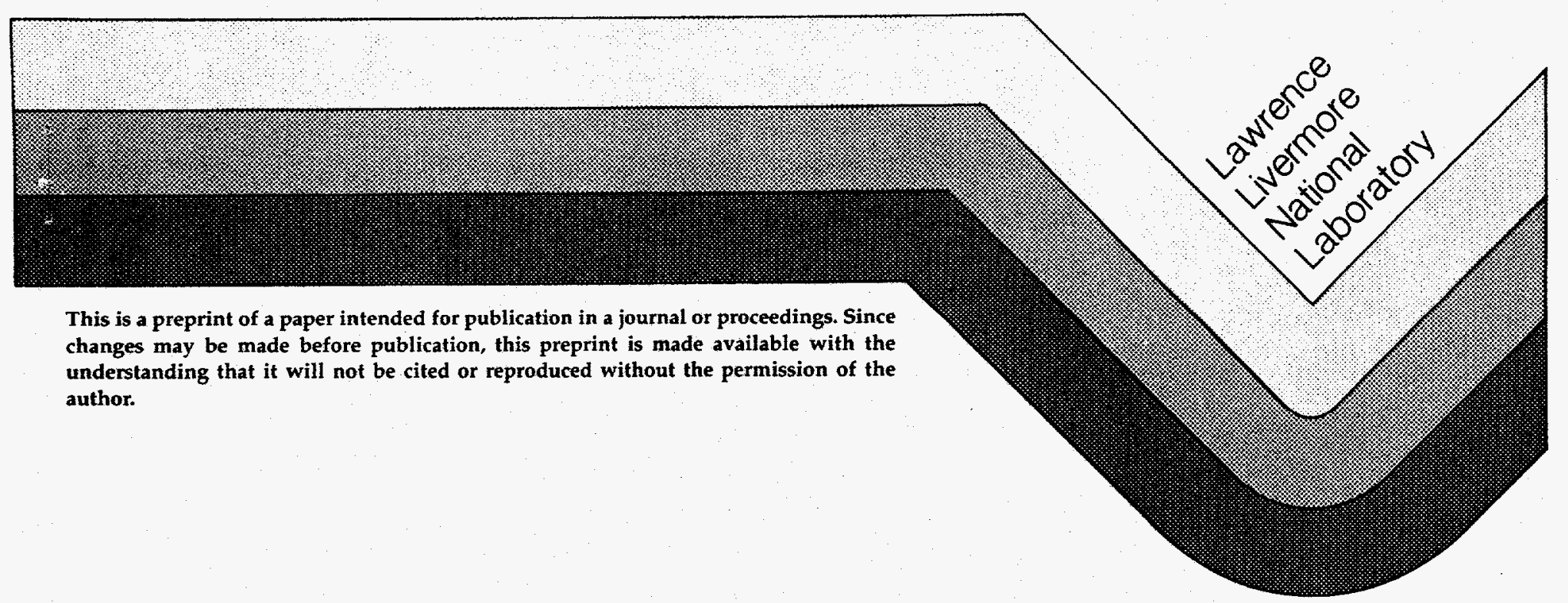




\title{
Nuclear Microscopy of Sperm \\ Cell Elemental Structure
}

Graham S. Bench1, Rod Balhorn ${ }^{2}$, Alexander M. Friz ${ }^{1}$ and S.P.H.T. Freeman 1

1.) Center for Accelerator Mass Spectrometry,

Lawrence Livermore National Laboratory,

Livermore, CA 94550

2.) Biology and Biotechnology Research Program, Lawrence Livermore National Laboratory,

Livermore, CA 94550

\begin{abstract}
Theories suggest there is a link between protamine concentrations in individual sperm and male fertility. Previously, biochemical analyses have used pooled samples containing millions of sperm to determine protamine concentrations. These methods have not been able to determine what percentage of morphologically normal sperm are biochemically defective and potentially infertile. Nuclear microscopy has been utilized to measure elemental profiles at the single sperm level. By measuring the amount of Phosphorus and Sulfur, the total DNA and protamine content in individual sperm from fertile bull and mouse semen have been determined. These values agree with results obtained from other biochemical analyses. Nuclear microscopy shows promise for measuring elemental profiles in the chromatin of individual sperm. The technique may be able to resolve theories regarding the importance of protamines to male fertility and identify biochemical defects responsible for certain types of male infertility.
\end{abstract}




\section{Introduction}

The biochemical composition and structure of an individual male sperm cell is extremely important because of the probability that it might fertilize an egg and provide the paternal DNA required for the development and growth of a fetus. The DNA is packed in a highly condensed state inside the nucleus of the sperm by two small proteins, protamine 1 and protamine 2 . The protamines are unique to sperm and their binding to DNA in the maturing sperm cell inactivates all the genes and 'deprograms' the genetic machinery in preparation for fertilization.

Biochemical analyses of human sperm obtained from fertile and infertile males have suggested that the relative proportion of protamine 1 and 2 bound to DNA is important for fertility. The sperm of fertile human males contain an equal amount of protamine 1 and $2[1,2]$. Sperm obtained from infertile individuals that only produce sperm with overly large, round heads (Round-headed sperm syndrome), on the other hand, have been shown to be deficient in protamine $2[1,2]$. Deficiencies in protamine 2 content have also been observed in other cases of human male infertility [3], with some individuals appearing to produce sperm lacking in protamine 2 altogether. Similar studies of the role protamines and other nuclear proteins play in the fertility of sperm from other species are also being undertaken [4].

While these studies have correlated protamine deficiencies in sperm with infertility, the conclusions have been drawn from analyses performed on whole semen or pools of millions of cells. In each case, the investigator could not rule out the possibility that the observed deficiency might reflect the presence of a sub-population of very abnormal sperm or spermatids arrested at a particular point in their development. Further, the prospect that a significant number of sperm produced by these infertile males may be biochemically normal could not be discounted. Microscopy studies have indicated that many infertile human males produce a small number of sperm that at least appear morphologically normal. The semen of normally fertile males

commonly contain $25-40 \%$ abnormal sperm, yet nothing is known about the biochemical composition of these abnormal cells. In order to properly study the biochemical composition of sub-populations of sperm it is essential that analyses be performed at the single cell level. 
At Livermore nuclear microscopy is being employed to quantitatively measure elemental concentrations in single sperm. Sperm are an ideal target for individual analysis by nuclear microscopy. Sperm heads are of size $\sim 8 \times 5 \mu \mathrm{m}^{2}$ and are $\sim 1 \mu \mathrm{m}$ thick and they can be easily isolated in large numbers. The heads of mature sperm consist of two thin membranes surrounding the nucleus and the nucleus occupies $98 \%$ of the volume of the sperm head. The DNA and protamines which fill the sperm nucleus are collectively termed sperm chromatin. Here, the use of nuclear microscopy to measure $\mathrm{P}$ and $\mathrm{S}$ concentrations in the chromatin of individual sperm obtained from fertile bull and mouse semen is reported. From these measurements the total protamine and DNA content per nucleus has been determined. These values agree with results obtained by other biochemical techniques.

\section{Method}

Mouse (Mus musculus) sperm were obtained from retired breeders of the FVB strain. This strain produces low levels of sperm with abnormal head morphology. Epididymal sperm were isolated from a pool of three mice. The mice were sacrificed by cervical dislocation following light anesthesia and the caudal portions of the epididymides were removed. Semen were released from the vas into $0.15 \mathrm{M}$ ammonium bicarbonate by applying pressure to each epididymis. Bull (Bos taurus) sperm were obtained from pooled semen (American Breeders Service, De Forest, Wisconsin) which had good fertility and low levels of sperm with abnormal head morphology. The semen was diluted in $0.15 \mathrm{M}$ ammonium bicarbonate. Diluted semen samples were centrifuged ( $5000 \mathrm{rpm}$ for 5 minutes) to pellet the sperm cells from the seminal fluid.

Microbeam PIXE spatial maps of individual freeze dried whole sperm (i.e. intact sperm with nuclear membranes and tails) indicated that some $S$ was contained in the tails and possibly the acrosome/nuclear membranes. In order to obtain accurate protamine and DNA values, these measurements indicated that it would be preferable to examine pure sperm chromatin free of contaminating nuclear membranes and tails. Two methods of isolating sperm chromatin from whole sperm were examined. In one, sperm were gently sonicated with a Teflon coated tip to 
break off the tails and disrupt the nuclear membranes. The solution was then centrifuged to selectively pellet the remaining heads which were then resuspended in $0.15 \mathrm{M}$ ammonium acetate. However, the sonication process, while being effective at removing the tails, did not appear consistent in the removal of the nuclear membranes. This method was deemed unsuitable.

In a second method sperm were treated with dithiothreitol and mixed alkyl trimethylammoniumbromide [5]. The solution was centrifuged to selectively pellet the sperm chromatin which were subsequently washed and resuspended in doubly distilled deionized water. It has been demonstrated that treatment of mammalian sperm by this process results in the complete removal and dissolution of the acrosome, nuclear membrane and tail of the sperm, leaving the chromatin intact and retaining the characteristic shape of the sperm nucleus [5]. The resuspended samples were then centrifuged to selectively pellet the sperm chromatin which were micro-pipetted onto ultra-clean, $1 \mu \mathrm{m}$ thick, transparent nylon foils stretched over a $15 \mathrm{~mm}$ diameter hole in a plastic support frame. The mounted samples were rapidly plunged in liquid nitrogen and freeze dried in a lyophilizer. The freeze dried samples were stored in a ultra-clean, sterile, dry environment prior to microprobe analysis. Optical studies revealed that the characteristic morphology of the nuclei were still retained. Further, $\mathrm{P}$ and $\mathrm{S}$ are tightly bound in sperm chromatin and are non-labile (sperm chromatin is insoluble in water and most buffers). It is unlikely that these components are lost during sample preparation. The data presented here are from samples prepared using this second method.

Chromatin from individual sperm were examined with the nuclear microprobe facility located at the Lawrence Livermore National Laboratory [6]. For each species only mature sperm that had the characteristic normal morphology were irradiated. Particle induced $x$-ray emission (PLXE) data were obtained using incident $3 \mathrm{MeV}$ proton microbeams. X-rays were detected with a $\mathrm{Si}(\mathrm{Li})$ detector that subtended a solid angle of $\sim 100 \mathrm{msr}$ at a scattering angle of $135^{\circ}$. Charge was collected in a biased Faraday cup located behind the sample. X-rays were recorded in time sequence along with coincident beam spatial co-ordinates arising from scanning the beam electrostatically over the sample in a point by point raster mode. 
For the measurements reported here, beam currents of up to $900 \mathrm{pA}$ focused down to spot sizes of $\sim 3 \mu \mathrm{m}$ were repeatedly scanned over areas of $\sim 23 \times 22 \mu \mathrm{m}^{2}$. Within each pass $0.1 \mathrm{nC}$ was deposited on each beam location. Areas were irradiated with an exposure of up to $1.5 \mu \mathrm{C}$. Each irradiated area contained the chromatin from a single sperm. Under these conditions $\mathrm{x}$-ray yields of both $\mathrm{P}$ and $\mathrm{S}$ remained constant throughout irradiation. However, if samples were irradiated with stationary unscanned microbeams elemental losses of $\mathrm{P}$ and $\mathrm{S}$ occurred for beam currents of a few nA. Others have also observed elemental losses during stationary beam spot irradiations and have also found that these losses can be minimized by scanning the beam [7].

Data were reduced off-line so that $\mathrm{x}$-ray spectra corresponding to the chromatin could be extracted from each irradiated region [8]. X-ray spectra were analyzed with the PDXEF spectrum fitting code [9]. $\mathrm{P}$ and $\mathrm{S}$ counting statistics from the chromatin of individual sperm were better than $3.5 \%$. Figure 1 shows an $\mathrm{x}$-ray spectrum from the chromatin of a single bull sperm. A series of thin film calibration standards containing Phosphorus and Sulfur were used to calibrate the $\mathrm{x}$-ray detection system. Scanning transmission ion microscopy measurements revealed that the sperm chromatin were $\sim 0.5 \mu \mathrm{m}$ thick. Hence, $\mathrm{P}$ and $\mathrm{S}$ masses (units of gram) in the chromatin of individual sperm were calculated using the thin film approximation, the total scan area and the exposure delivered to the total scan area. The area occupied by the chromatin factors out in these calculations. The measurement of $\mathrm{P}$ and $\mathrm{S}$ masses has a quantitative accuracy of $<$ $7 \%$ ( $4 \%$ detector efficiency/charge collection and $5 \%$ scan area calibration added in quadrature).

\section{Results and Discussion}

From the $\mathrm{P}$ and $\mathrm{S}$ masses, the DNA and protamine content in a single sperm can be derived. Sperm chromatin primarily consists ( $>99 \%$ ) of DNA and protamines. The Phosphorus content of sperm chromatin provides an accurate measure of the DNA content (sperm do not contain RNA and protamines do not contain Phosphorus). A DNA base pair has a molecular weight of $660 \mathrm{~g} / \mathrm{Mol}$ and contains 2 Phosphorus atoms but no Sulfur. The molecular weight of Phosphorus is $31 \mathrm{~g} / \mathrm{Mol}$. To obtain the DNA mass in sperm chromatin the Phosphorus mass is multiplied by 
$(660 /(2 \times 31))$. Because the protamines which constitute $97-99 \%$ of the protein in chromatin contain a known number of cysteine residues (the only Sulfur containing amino acid in bull and mouse protamine) per molecule, Sulfur should be an effective measure of the protamine content.

Bull sperm only contain protamine 1 which has a molecular weight of $6627 \mathrm{~g} / \mathrm{Mol}$ and contains 7 Sulfur atoms. Sulfur has a molecular weight of $32 \mathrm{~g} / \mathrm{Mol}$. To obtain the amount of protamine 1 in bull sperm chromatin, the Sulfur mass is multiplied by $(6627 /(7 \times 32))$. Mouse sperm contains both protamine 1 and protamine 2 . The mass ratio of protamine 1 to protamine 2 in fertile mouse sperm is $0.33 \pm 0.02$ [10]. Mouse protamine 1 has a molecular weight of 6827 $\mathrm{g} / \mathrm{Mol}$ and contains 9 Sulfur atoms while mouse protamine 2 has a molecular weight of 8594 $\mathrm{g} / \mathrm{Mol}$ and contains 7 Sulfur atoms. To obtain the total amount of protamine in fertile mouse sperm the Sulfur mass is multiplied by

$$
\frac{8594 \times 2}{7 \times 32 \times 3}+\frac{6827}{9 \times 32 \times 3}=33.5
$$

A total of 19 bull sperm and 20 mouse sperm have been analyzed and the results are shown in table 1. The DNA measurements agree with DNA measurements determined by Phosphorus analysis, deoxyribose analysis, and absorption spectroscopy at $260 \mathrm{~nm}$ using pools of sperm [10] and DNA measurements performed on individual sperm using cytomorphometry and cytospectrophotometry $[10,11]$. These previous analyses indicate that both fertile bull and mouse sperm contain $\sim 3.3 \mathrm{pg}$. DNA/nucleus (ranging from 3.1 to $3.6 \mathrm{pg}$.). The mouse protamine value of $2.87 \pm 0.15 \mathrm{pg}$. is in agreement with total protamine measurements determined by quantitative amino acid analysis, absorption spectroscopy at $230 \mathrm{~nm}$ and sperm head density analysis in Cesium Chloride. These pooled sample analysis techniques yield a chromatin protein content of $\sim 3.0 \mathrm{pg}$. per nucleus (ranging from 2.8 to $3.2 \mathrm{pg}$.) for mouse sperm [10]. Values have not been published for the protein content of bull sperm, however the mass ratio of protamine to DNA in fertile bull sperm is believed to be $\sim 0.9$. For this work the mass ratio of protamine to DNA in individual bull sperm chromatin was $0.85 \pm 0.04$ while in mouse sperm chromatin it was $0.85 \pm 0.06$ (results are expressed as mean \pm standard deviation). 
The standard deviations in the protamine and DNA data presented here are 3 to $7 \%$ of the mean values demonstrating that $10 \%$ differences in both DNA and protamine content should be detectable in male infertility studies with the use of nuclear microscopy.

These results are of interest because they demonstrate that this technique can be used to accurately measure the total protamine content of individual sperm cells. At present, this cannot be achieved with other biochemical methods. The technique should be able to facilitate the analysis of sperm sub-populations. For instance it may be possible to determine whether abnormally shaped sperm in fertile semen contain the proper amount of protamine. Further, individual sperm from infertile males suspected to have protamine deficiencies could be examined. It may be possible to determine whether a) the majority of sperm produced by infertile individuals are deficient in protamine, and $b$ ) these males produce a sub-population of the sperm that appear biochemically normal. It may be also be possible to monitor the expression of normal and mutant genes in transgenic mice in studies investigating protamine function.

\section{Conclusion}

Nuclear microscopy has been employed to determine the amount of Phosphorus and Sulfur in the chromatin of individual sperm from fertile bull and mouse semen. From these data the total DNA and protamine content in individual nuclei have been determined. These values agree with results obtained by biochemical analyses performed on pools of millions of sperm and/or staining techniques performed on individual sperm. The technique may be able to resolve theories regarding the importance of protamines to male fertility, identify biochemical defects responsible for certain types of male infertility and determine features of sperm chromatin structure.

*This work was performed under the auspices of the U.S. Department of Energy by Lawrence Livermore National Laboratory under contract No. W-7405-Eng-48. 


\section{References}

[1] Balhorn, R., Reed, S. and Tanphaichitr, N., (1988), Experientia, 44:52.

[2] Belokopytova, I.A., Kostyleva, E.I., Tomilin, A.N. and Vorobev, V.I., (1993), Mol. Reprod. Devel. 34:53.

[3] de Yebra, L., Ballesca, J.L., Vanrell, J.A., Bassas, L. and Oliva, R., (1993), J. Anal. Chem., 268:10553.

[4] Love, C.C., (1993), Ph.D. Thesis, University of Pennsylvania, U.S.A.

[5] Balhorn, R., Gledhill, B.L., and Wyobrek, A.J., (1977), J. Biochemistry, 16:4074.

[6] Roberts, M.L., Bench, G.S., Heikkinen, D.W., Morse, D.H., Bach, P., Pontau, A.E., and Antolak, A.J., submitted to Proceedings of the Fourth International Conference on Nuclear Microprobe Technology and Applications, Shanghai, China, October 1994.

[7] Legge, G.J.F., and Mazzolini, A.P., (1980), Nucl. Instr. and Meth., 168:139.

[8] Antolak, A.J., Bench, G.S. and Morse, D.H., (1994), Nucl. Instr. and Meth., B85:597.

[9] Antolak, A.J. and Bench, G.S., (1994), Nucl. Instr. Meth., B90:596.

[10] Pogany, G.C., Corzett, M., Weston, S. and Balhorn, R., (1981), Exp. Cell. Res., 136:127.

[11] Roberts, B. and Roberts, S., (1972), Chromosoma, 39:83.

\section{Figure Captions.}

Figure 1: PIXE spectrum of the chromatin from a single bull sperm. The dotted line corresponds to the raw data, the dashed line is the background estimation, while the solid line shows the fit to the $\mathrm{x}$-ray peaks. The spectrum was formed from a dose of $0.24 \mu \mathrm{C}$ to the chromatin. Prominent $\mathrm{x}$-ray lines are labeled.

Table 1: Average DNA and protamine content in bull and mouse sperm chromatin together with the number of sperm examined in each species. Results are expressed as mean \pm standard deviation. The minimum and maximum values of DNA and protamine content within single sperm of each species are also shown. 
Table 1:

\begin{tabular}{|c|c|c|c|c|c|}
\hline $\begin{array}{c}\text { Type } \\
\text { of sperm }\end{array}$ & $\begin{array}{c}\text { Number } \\
\text { analyzed }\end{array}$ & $\begin{array}{c}\text { DNA } \\
\text { content (pg.) }\end{array}$ & $\begin{array}{c}\text { Minimum and } \\
\text { maximum DNA } \\
\text { content (pg.) }\end{array}$ & $\begin{array}{c}\text { Protamine } \\
\text { content (pg.) }\end{array}$ & $\begin{array}{c}\text { Minimum and } \\
\text { maximum protamine } \\
\text { content (pg.) }\end{array}$ \\
\hline bull & 19 & $3.42 \pm 0.09$ & $3.24,3.56$ & $2.90 \pm 0.12$ & $2.67,3.09$ \\
\hline mouse & 20 & $3.36 \pm 0.09$ & $3.22,3.59$ & $2.87 \pm 0.15$ & $2.67,3.13$ \\
\hline
\end{tabular}




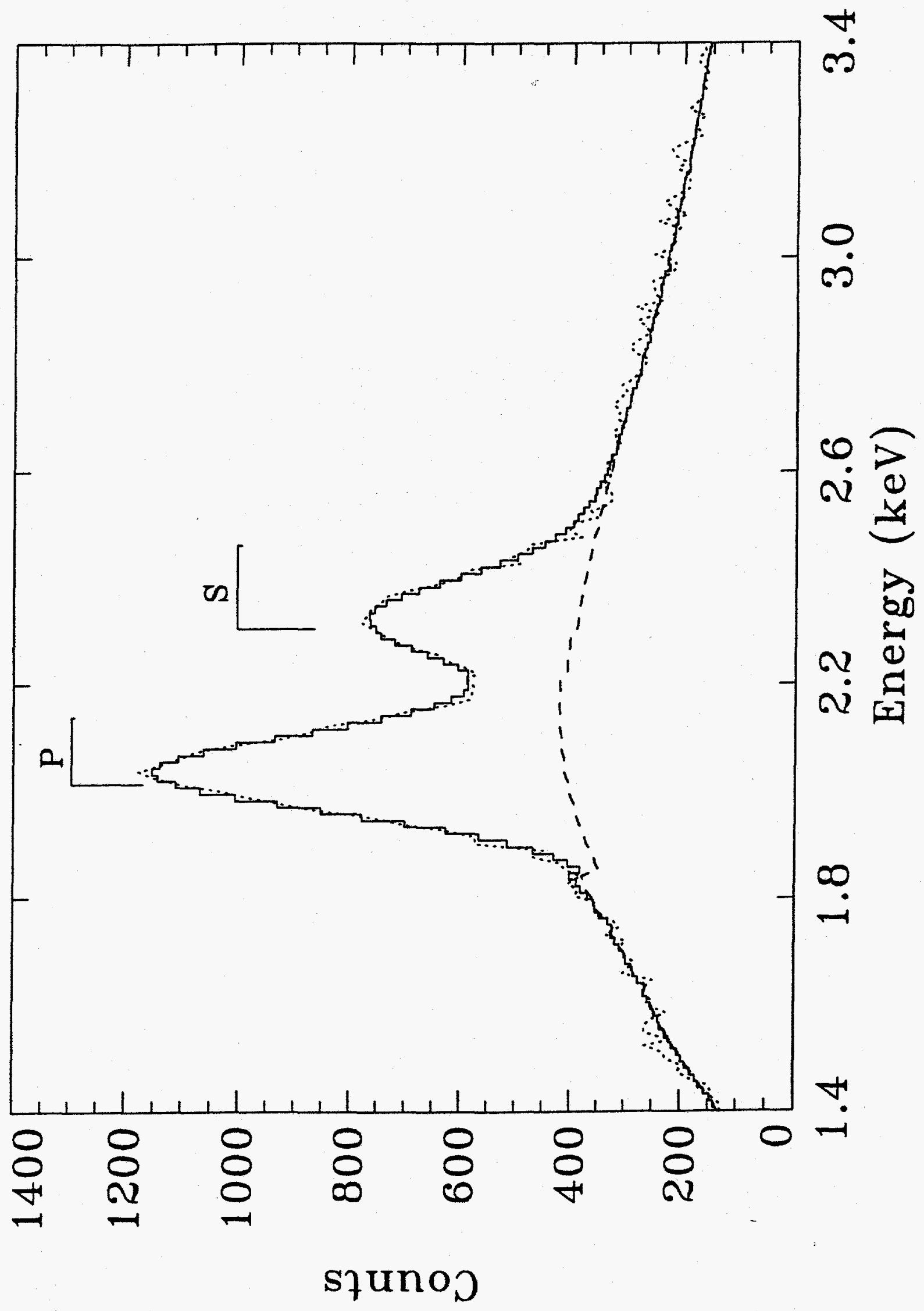

